\title{
A dual padding method for ischial pressure sore reconstruction with an inferior gluteal artery perforator fasciocutaneous flap and a split inferior gluteus maximus muscle flap
}

\author{
Inhoe $\mathrm{Ku}^{1}$, Gordon K. Lee ${ }^{2}$, Saehoon Yoon ${ }^{3}$, Euicheol Jeong ${ }^{3}$ \\ ${ }^{1}$ Department of Plastic and Reconstructive Surgery, Seoul National University College of Medicine, Seoul, Korea; ${ }^{2}$ Division of Plastic and \\ Reconstructive Surgery, Stanford University Medical Center, Stanford, CA, USA; ${ }^{3}$ Department of Plastic Surgery, SMG-SNU Boramae \\ Medical Center, Seoul, Korea
}

Background Various surgical management methods have been proposed for ischial sore reconstruction, yet it has the highest recurrence rate of all pressure ulcer types. A novel approach combining the advantages of a perforator-based fasciocutaneous flap and a muscle flap is expected to resolve the disadvantages of previously introduced surgical methods.

Methods Fifteen patients with ischial pressure ulcers with chronic osteomyelitis or bursitis, who underwent reconstructive procedures with an inferior gluteal artery perforator (IGAP) fasciocutaneous flap and a split inferior gluteus maximus muscle flap from January 2011 to June 2016, were analyzed retrospectively. The split muscle flap was rotated to obliterate the deep ischial defect, managing the osteomyelitis or bursitis, and the IGAP fasciocutaneous flap was rotated or advanced to cover the superficial layer. The patients' age, sex, presence of bursitis or osteomyelitis, surgical details, complications, follow-up period, and ischial sore recurrence were reviewed.

Results All ischial pressure ulcers were successfully reconstructed without any flap loss. The mean duration of follow-up was 12.9 months (range, 3-35 months). Of 15 patients, one had a recurrent ulcer 10 months postoperatively, which was repaired by re-advancing the previously elevated fasciocutaneous flap.

Conclusions The dual-flap procedure with an IGAP fasciocutaneous flap and split inferior gluteus maximus muscle flap for ischial pressure ulcer reconstruction is a useful method that combines the useful characteristics of perforator and muscle flaps, providing thick dual padding with sufficient vascularization while minimizing donor morbidity and vascular pedicle injury.

Keywords Pressure ulcer / Surgical flaps / Osteomyelitis / Bursitis / Ischium
Correspondence: Euicheol Jeong Department of Plastic Surgery, SMGSNU Boramae Medical Center, 20 Boramae-ro 5-gil, Dongjak-gu, Seoul 07061, Korea

Tel: +82-2-870-2331

Fax: $+82-2-831-2826$

E-mail: ecjeong@snu.ac.kr
This article was presented at the PRS Korea 2016 on November 17-20, 2016, in Seoul, Korea.

\section{INTRODUCTION}

A pressure ulcer is a chronic wound with physiologically im- paired healing induced by prolonged pressure applied over a skin lesion. Patients with a neurological deficit or spinal injury with paraplegia and quadriplegia are vulnerable to pressure ul- 
cers due to sensory loss. Pressure ulcers are frequently found superficial to bony prominences, where pressure is applied directly on the soft tissue, causing ischemic damage. The occurrence of ischial ulcers is associated with the pressure points on the ischial tuberosities. When a patient maintains a sitting position, the resultant shearing force increases the risk for developing an ulcer, and this occurs in patients who are confined to a wheelchair.

Surgical procedures for treating ischial ulcers should consider the likelihood of relapse of the ulcer, meaning that surgeons must utilize a comprehensive approach to preserve vascular pedicles for the potential elevation of other optional flaps in order to reconstruct recurrent ischial ulcers or sores at other sites. Flaps for ischial ulcer reconstruction should be well-vascularized to promote survival, to facilitate infection control, and to provide adequate size and bulkiness to obliterate dead space.

Various methods have been proposed for reconstruction flaps. As a representative overview, V-Y advancement of the hamstring muscles; inferior gluteus maximus, gracilis, and rectus abdominis muscle flaps; and gluteus thigh fasciocutaneous flaps have been utilized for ischial ulcers [1-4]. However, no single surgical technique has been proven effective over others. Subsequently, advances in the understanding of perforators led to the use of perforator flaps for ischial reconstruction. Currently, classical musculocutaneous flaps and the inferior gluteal artery perforator (IGAP) fasciocutaneous flap are frequently used, with continuous modifications to find innovative and effective ways to obtain better outcomes.

Therefore, we present a method of integrating the advantages of a perforator-based fasciocutaneous flap and a muscle flap for ischial ulcer reconstruction through adjoining the gluteus maximus muscle flap and IGAP fasciocutaneous flap together. The gluteus maximus muscle is split, and its inferior portion is used. This dual padding can provide bulkiness to occupy the dead space and double the layer thickness, helping to prevent pressure ulcer recurrence. In dual-flap coverage procedures, the discrepancy of the defect's anatomical location in terms of depth is also resolved by transposing each flap into an adequate position.

\section{METHODS}

The study was conducted between 2010 and 2016 among patients with grade IV ischial pressure ulcer with either ischiogluteal bursitis or osteomyelitis. Fifteen patients underwent pressure ulcer surgical reconstruction with the combined method of a split inferior gluteus muscle flap and an IGAP fasciocutaneous flap. Thirteen patients were men, and two patients were women, with a mean age of 53.7 years (range, 28-67 years). Of the 15 patients, 14 had paraplegia and one had paraparesis, with etiolo- gies of spinal injury and spinal infection in 14 and one patient, respectively. Detailed information regarding the patients is shown in Table 1 . This study was approved by our institutional ethics committee, and informed written consent was obtained from patients.

Under general anesthesia, surgery was performed with the patient lying in the prone position with both hip joints flexed to flatten the gluteal fold. Complete debridement of the necrotic tissue and marginal scar over the ischial sore was performed, and the ischiogluteal bursa was excised. Tissue culture was performed in every case, and partial ostectomy was performed until bone bleeding was identifiable if osteomyelitis of the ischium was clinically and radiographically diagnosed.

To outline the gluteus maximus muscle, a line between the coccyx, posterior superior iliac spine (PSIS), and lateral border of the greater trochanter was drawn. Another vertical line was drawn from the PSIS to the gluteal fold to identify IGAPs for IGAP fasciocutaneous flap elevation. Using a handheld Doppler flowmeter, the perforators were mostly found in the middle third of the line. The IGAPs for preservation were chosen from skin areas where there was no scarring or infection. The flap was designed to be a maximum of $10 \times 20 \mathrm{~cm}$, considering the defect and arc of rotation. At least two perforators were preserved to secure the viability of the flap. The horizontal axis of the flap was designed to be nearly as long as the gluteal crease (Fig. 1A). After incising the superior and inferior border of the IGAP fasciocutaneous flap, dissection was performed through beveling from the skin to the muscle. The IGAP fasciocutaneous flap was elevated from the distal region. The IGAP was not skeletonized, and the surrounding soft tissues were preserved. Sufficient mobility of the flap was obtained by detaching the flap from the gluteus muscle. Elevation of the IGAP fasciocutaneous flap exposed the distal insertion portion of the gluteus maximus muscle, and the inferior border of the muscle was identified. After estimating the defect size and the arc of rotation, the inferior portion of the gluteus maximus muscle was detached from the insertion site to the great trochanter, and dissection was performed parallel to the muscle fiber to raise the flap. Although the color of the gluteus maximus muscle was altered and resembled the color of fat, the presence of the usual muscle fibers meant that it was not difficult to identify. The inferior medial portion of the flap was preserved. Although the patients had experienced long periods of paraplegia, the size of the gluteus maximus muscle remained sufficient to identify and use it after splitting. Subsequently, the split muscle flap was rotated to fill the dead space, which was created by ischial ulcer removal and ostectomy (Fig. 1B). The flap was fixed to the remnant muscle fascia or periosteum with 2-0 Vicryl sutures. Subsequently, the 


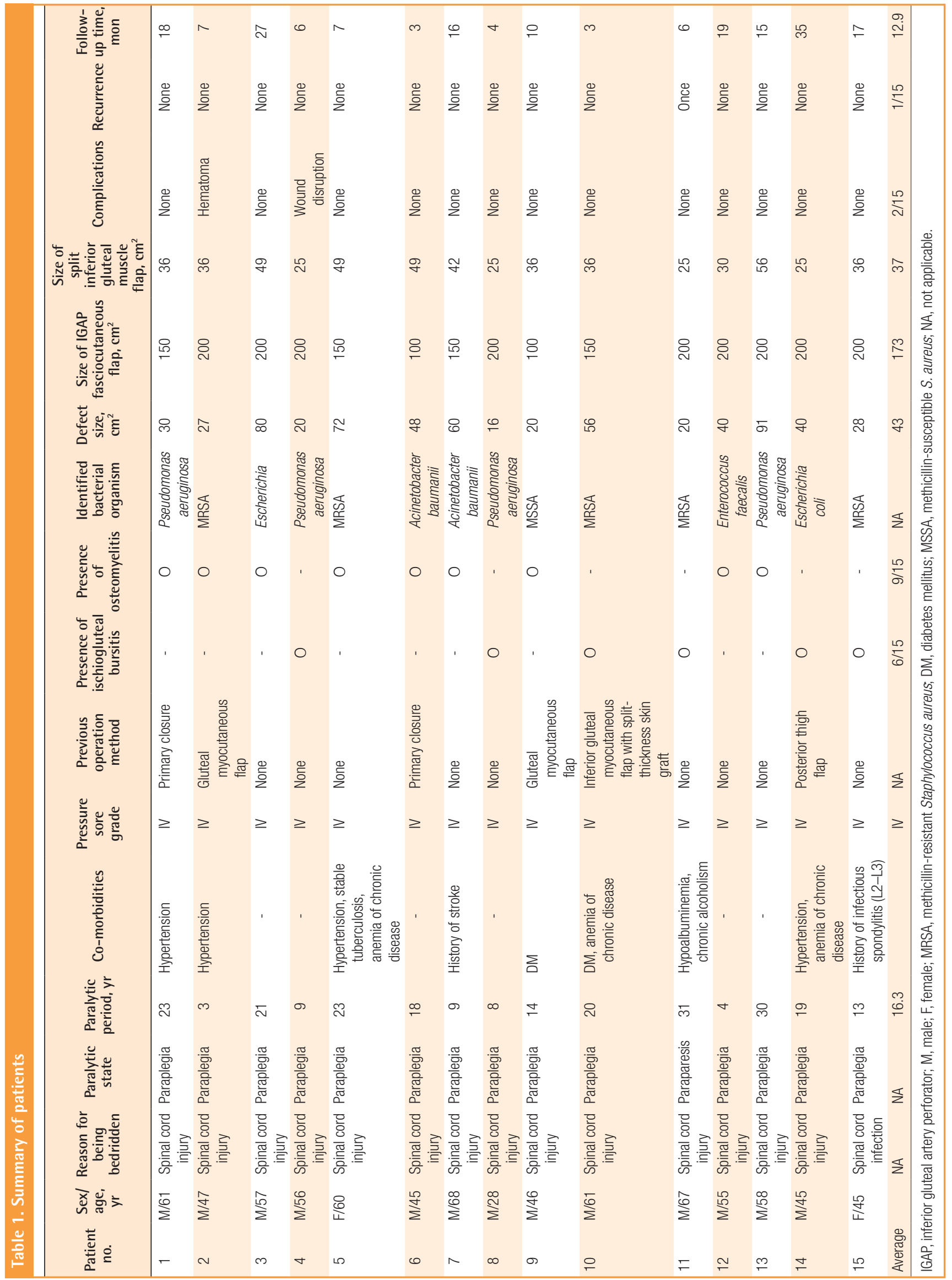




\section{Fig. 1. The IGAP and muscle flaps design}

(A) To locate the inferior gluteal artery perforators (IGAPS), a perpendicular line was drawn from the posterior superior iliac spine (PSIS) to the medial gluteal fold, and IGAPs were usually found around the middle third of the line. (B) In setting of the split gluteus maximus muscle flap and IGAP flap.
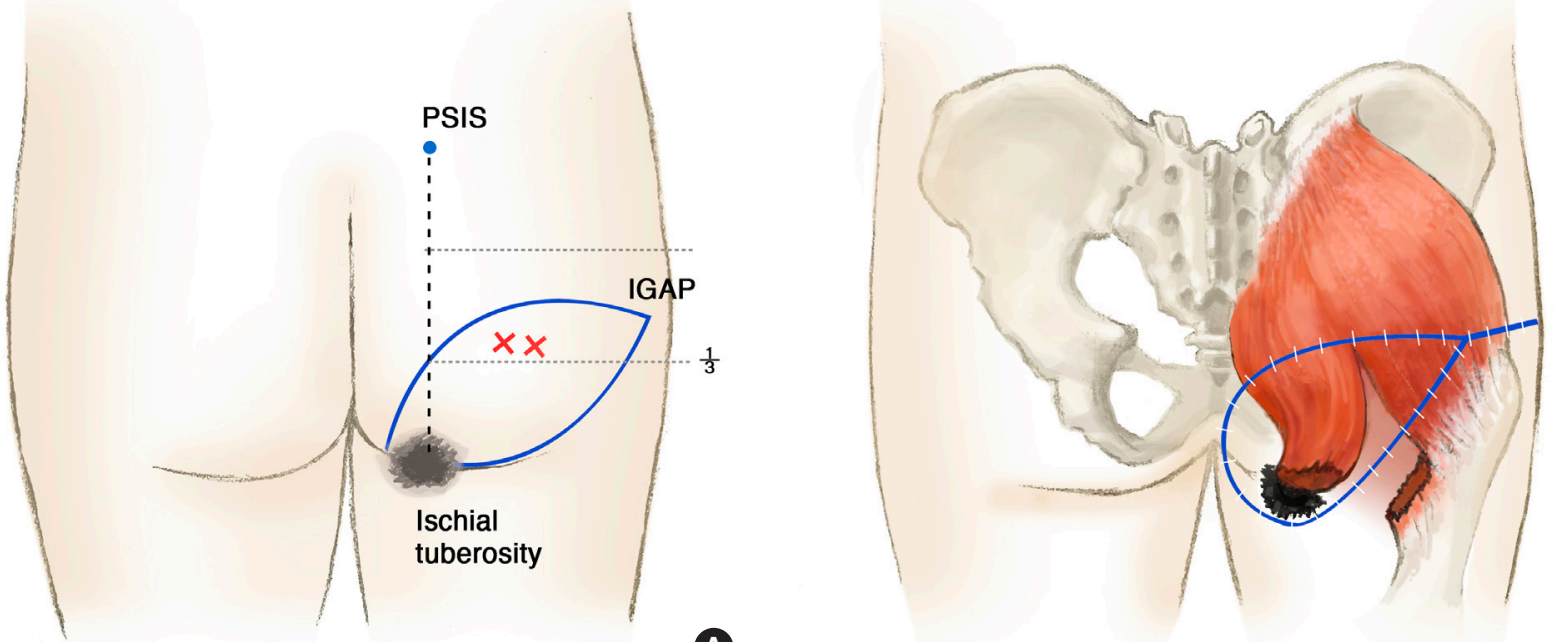

A

B

\section{Fig. 2. Moderate ischial pressure near the anus}

The fasciocutaneous flap was rotated $90^{\circ}$ and inset near the anus, and the split gluteus maximus muscle flap was used to fill the cavity after removal of tissue affected by bursitis. (A) Ischial sore near the anus in a female patient. (B) Isolated inferior gluteal artery perforator (IGAP)-based fasciocutaneous flap. (C) Splitting the gluteus maximus muscle as a flap. (D) Split gluteus maximus muscle flap rotated on the ischial tuberosity. (E) IGAP fasciocutaneous flap rotated $90^{\circ}$ over the split muscle flap. (F) One-year follow-up photograph.
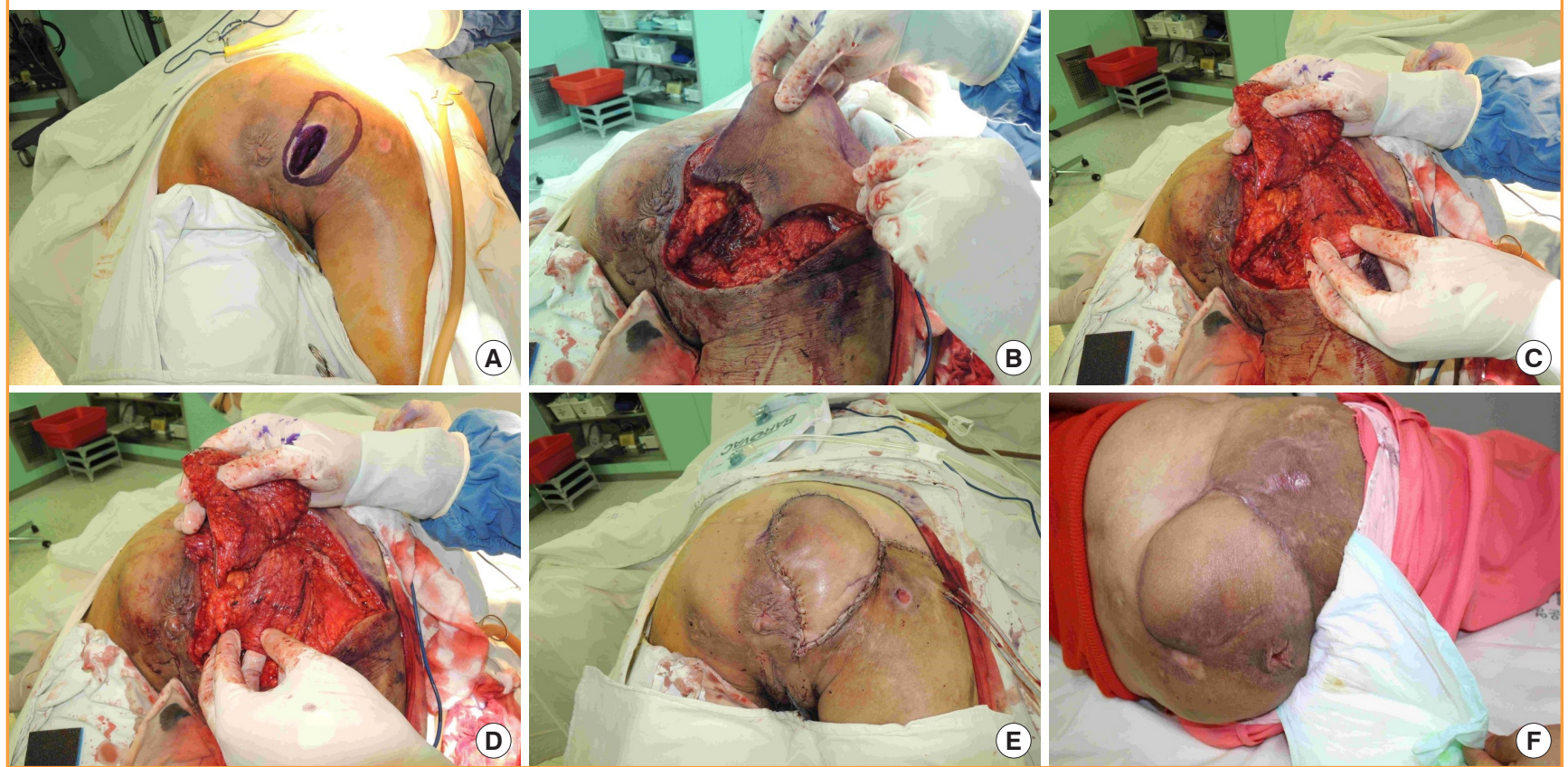

previously elevated IGAP fasciocutaneous flap was advanced or rotated to cover the transposed muscle flap. If the skin defect was located close to the anus, $90^{\circ}$ of rotation was preferred rath- er than advancement of the flap. The donor site was closed primarily (Fig. 2).

During the postoperative period, patients maintained a prone 


\section{Fig. 3. A recurrent case after surgery}

Re-advancement of only the inferior gluteal artery perforator (IGAP) flap was an acceptable option. (A) A skin incision was made at the previous flap incision wound and IGAP fasciocutaneous flap elevation at the subfascial layer was achieved. (B) A flap that was based on the IGAPs preserved in the previous operation was elevated and advanced to repair the defect.

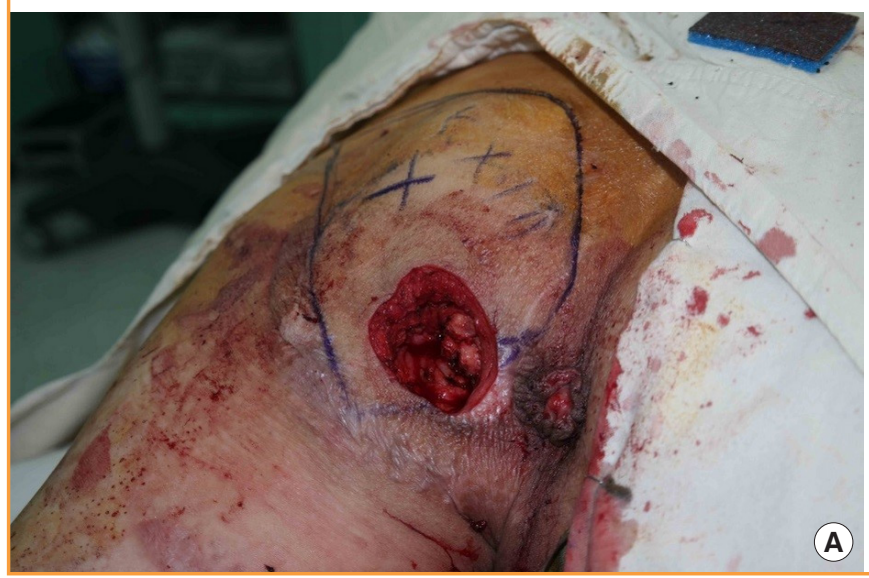

position to avoid pressure and shearing force upon the reconstructed area. For 2 weeks, positional changes to the supine position or ambulation were avoided as much as possible to minimize the occurrence of wound complications, including hematoma, seroma formation, and wound dehiscence. We inspected the wound daily while performing dressing, and intravenous antibiotics were used for 6 weeks based on the organisms isolated from the infected tissue obtained intraoperatively.

\section{RESULTS}

All patients had combined bursitis or osteomyelitis with bacterial organisms isolated from the bursa and sequestrum. Six patients had recurrent cases of ischial ulcer from previous operations. Of the 15 patients, all ischial ulcer cases had successful reconstruction without any partial or total loss of the flap. One case of wound dehiscence was observed on the third day after suture removal in the outpatient clinic, and was repaired immediately. One case of hematoma was noted on the third postoperative day. It was managed with surgical drainage and reapplication of closed suction drainage. One recurrent ischial ulcer was identified 10 months postoperatively. The previously elevated IGAP fasciocutaneous flap was re-advanced to close the defect (Fig. 3).

\section{DISCUSSION}

Ischial pressure ulcers are most commonly observed in paraplegic patients in wheelchairs, wherein the tissue pressure reaches 80 to $100 \mathrm{mmHg}$ depending on their anatomic position [5]. The location of the ischium near the perineal region makes the

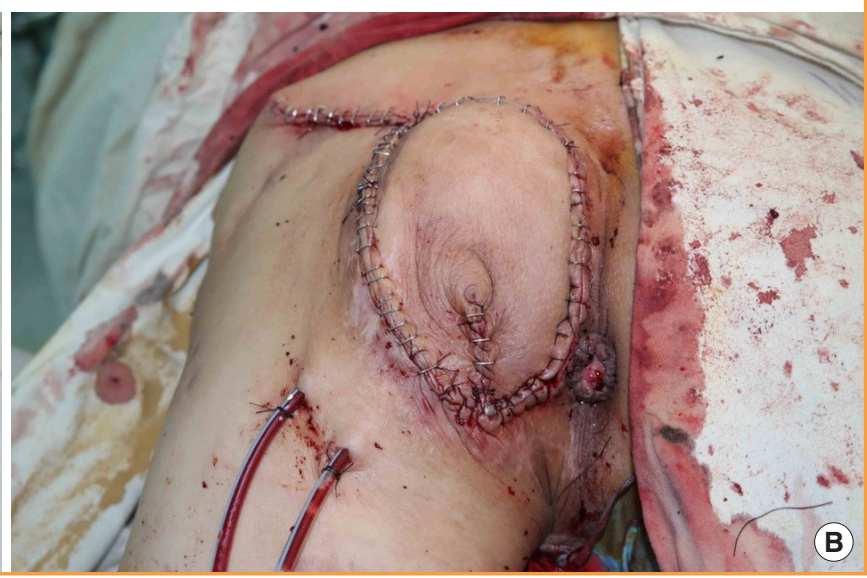

ischial region prone to contamination by feces and urine, leading to skin maceration and persistent infection [6,7]. Chronic infection of a lesion triggers osteomyelitis of the ischial tuberosity and ischiogluteal bursitis accompanied with necrotic soft tissue. Necrotic tissue should be debrided, after which an empty pocket that lacks soft tissue at the ischial area is required for flap reconstruction. In conservative approaches, the bony prominence at the ischium should be flattened to lower the local pressure, and the dead space must be obliterated.

A potential problematic point of surgical care for ischial ulcers is that the skin ulcers may not form directly on the vertical aspect of the region affected by osteomyelitis or bursitis, depending on the patient's wheelchair-bound posture [8]. In our study, the opening of the ischial ulcers was located near the perineum or superior-medial side of posterior thigh and gradually progressed to the ischial tuberosity, and the deep pockets following debridement were beveled up to the skin. Opening of ischial pressure sores was not observed on the vertical aspect of the ischial tuberosity at the anatomical position. The patients' sitting posture applies pressure on the bony prominence of the ischial tuberosity and the soft tissue located above, causing vertically disproportional ulcerative changes in the overlying tissue under the anatomical position. Separate flap coverage for deep and superficial areas of the defect has the favorable effect of reconstructing the soft tissue defect at its exact location, which is desirable, and the dual-flap technique also enables the reconstruction of the soft tissue layer-by-layer with the advantages each flap $[9,10]$.

Fasciocutaneous flaps provide higher mechanical resistance than muscle flaps, making them more suitable for reconstructing the superficial layer, while muscle flaps have reliable blood 
supply and adequate durability for preventing osteomyelitis in the deep layer. A split gluteus maximus muscle flap and an IGAP fasciocutaneous flap are ideal choices for the dual-flap coverage of ischial sores. The use of this combination distributes the pressure over two different flaps, thereby increasing the flap survival rate [11].

The gluteus maximus muscle flap is a well-defined and reliable flap that provides bulkiness over the buttocks. This well-vascularized mobilized muscle flap maintains a sufficient blood supply to control infected tissue and hypoxia caused by the mechanical stress imposed by patients' constant sitting position. Splitting the muscle increases the mobility of the flap and increases its volume for filling the defect by providing a degree of freedom. In our study, $30 \%-40 \%$ of the gluteus maximus muscle was detached from its caudal femoral insertion site for flap transposition. The muscle flap could be isolated on the avascular plane over the ischial insertions of the semitendinosus and semimembranosus muscles. In this way, the muscle flap could fill defects as large as $91 \mathrm{~cm}^{2}$. Because the elevation of a split gluteus maximus flap does not require additional skin incision, the vascular pedicle of the muscle is preserved so that the flap can be re-advanced if the ischial pressure ulcer recurs, and the undetached portion of the gluteus maximus muscle, which is helpful to prevent decubitus ulcers, can be used if a sacral ulcer occurs in the future $[12,13]$. Sacrificing the inferior part of the gluteus maximus muscle incurs slight mobility, but the other part of the muscle is able to maintain its function with the aid of other adductors $[2,14]$. The preservation of the insertion and origin of the gluteus maximus muscle in our procedures means that ambulatory function would not be compromised.

The IGAP-based fasciocutaneous flap is renowned for providing well-vascularized skin with a large angiosome, and it was previously reported to be able to cover ischial ulcers alone $[15,16]$. The large extent of the blood supply area of the flap permitted its use to reconstruct the superficial layer of pressure ulcers. An anatomical study showed that the mean area supplied by the IGAP is $22.1 \times 10.2 \mathrm{~cm}$, and the number of perforators is 7 to 14 [17]. In our case series, we were able to save at least two perforators without intramuscular dissection, and the flap was elevated subfascially to as large as $10 \times 20 \mathrm{~cm}$. The remaining perforators were ligated to increase the mobility of the flap, which is a merit of a perforator-based fasciocutaneous flap. It could be rotated $90^{\circ}$ or horizontally advanced for defect closure even near the anus. In two of the 15 cases of surgical treatment IGAP fasciocutaneous flap rotation was to cover the soft tissue defect of the ischial pressure sore. No cases of flap congestion or partial necrosis occurred, because we preserved at least two perforators. However, we suspect that flap rotation of $180^{\circ}$ may have the potential to cause flap congestion or deterioration by kinking the two or more perforators. Multiple perforators were preserved to make a reserve option for re-advancement if needed to treat recurrence. In our study, an ischial pressure ulcer recurred in one case, and revisional surgery was performed through the straightforward advancement of the previously rotated IGAP fasciocutaneous flap. While performing a split gluteus maximus muscle flap and an IGAP fasciocutaneous flap together, other adjacent muscles (except the gluteus maximus) were not interrupted, as they could serve as potential donor sites of a flap for a recurrent ischial pressure ulcer. The donor site for the IGAP fasciocutaneous flap could be primarily closed without tension.

The dual padding provided by the combination of a split gluteus maximus flap and an IGAP fasciocutaneous flap minimized recurrence, as one case of recurrence was observed during a 12.9-month follow-up period in 15 patients. In contrast, previous studies on the coverage of ischial pressure ulcers have reported significant recurrence rates ( $8 \%-64 \%$ depending on the series), and this discrepancy may be indicative of the usefulness of this method [13]. Additionally, a previous report of ischial pressure ulcer reconstruction with an IGAP fasciocutaneous flap alone demonstrated five recurrent cases among 23 patients within a 25.4-month follow-up period, corresponding to a higher recurrence rate than was observed using the dual padding method [16].

This technique has a few drawbacks, including potential limitations in elevating and transposing both an IGAP-based fasciocutaneous flap and a split gluteus maximus muscle flap in patients with multiple pressure ulcers who have undergone previous operations at the flap donor site, resulting in a lack of fresh soft tissue. In this study, we encountered two patients who had previously received a gluteus maximus myocutaneous flap. Fortunately, their surgical histories were very clear, and it was possible to utilize the dual-flap technique and harvest a large myocutaneous flap without dissection to search for musculocutaneous perforators. For patients with a previous history of surgery on the buttocks, preoperative computed tomography angiography and Doppler flowmetry to confirm the anatomical status of the IGAP are recommended.

The operation time could not be compared because relevant data could not be found in the literature. In the authors' experience, raising the two different flaps meaningfully increased the operation time compared to raising an IGAP fasciocutaneous flap alone. Nevertheless, the advantages of dual-flap coverage for ischial pressure sore reconstruction imply that it may be useful for further applications and worthy of additional discussion among plastic and reconstructive surgeons. 
In conclusion, the combination of an IGAP fasciocutaneous flap with a split gluteus maximus muscle flap resulted in high success rates for ischial pressure ulcer coverage in grade IV sores. For the management of recurrent ischial ulcers, adequate wound debridement should be performed and re-advancement of fasciocutaneous flaps can be attempted. This combination is worth considering in the management of patients with a deep ischial pressure ulcer.

\section{NOTES}

\section{Conflict of interest}

No potential conflict of interest relevant to this article was reported.

\section{Ethical approval}

The study was approved by the Institutional Review Board of SMG-SNU Boramae Medical Center (IRB No. 16-2016-85) and performed in accordance with the principles of the Declaration of Helsinki. Written informed consents were obtained.

\section{Patient consent}

The patients provided written informed consent for the publication and the use of their images.

\section{Author contribution}

Conceptualization: Jeong E, Lee GK. Data curation: Jeong E, Yoon S. Formal analysis: Jeong E, Ku I. Methodology: Jeong E. Project administration: Jeong E. Visualization: Ku I. Writing original draft: Ku I. Writing - review \& editing: Jeong E, Lee GK. Approval of final manuscript: all authors.

\section{ORCID}

Inhoe Ku https://orcid.org/0000-0002-3046-6800

Gordon K. Lee https://orcid.org/0000-0002-0093-9160

Euicheol Jeong https://orcid.org/0000-0003-2434-875X

\section{REFERENCES}

1. Hurteau JE, Bostwick J, Nahai F, et al. V-Y advancement of hamstring musculocuataneous flap for coverage of ischial pressure sores. Plast Reconstr Surg 1981;68:539-42.

2. Ramirez OM, Orlando JC, Hurwitz DJ. The sliding gluteus maximus myocutaneous flap: its relevance in ambulatory patients. Plast Reconstr Surg 1984;74:68-75.

3. Pena MM, Drew GS, Smith SJ, et al. The inferiorly based rectus abdominis myocutaneous flap for reconstruction of recurrent pressure sores. Plast Reconstr Surg 1992;89:90-5.

4. Rubin JA, Whetzel TP, Stevenson TR. The posterior thigh fasciocutaneous flap: vascular anatomy and clinical application. Plast Reconstr Surg 1995;95:1228-39.

5. Lindan O, Greenway RM, Piazza JM. Pressure distribution on the surface of the human body. I. Evaluation in lying and sitting positions using a "bed of springs and nails". Arch Phys Med Rehabil 1965;46:378-85.

6. Resnick NM, Beckett LA, Branch LG, et al. Short-term variability of self report of incontinence in older persons. J Am Geriatr Soc 1994;42:202-7.

7. Chassagne P, Landrin I, Neveu C, et al. Fecal incontinence in the institutionalized elderly: incidence, risk factors, and prognosis. Am J Med 1999; 106:185-90.

8. Tam EW, Mak AF, Lam WN, et al. Pelvic movement and interface pressure distribution during manual wheelchair propulsion. Arch Phys Med Rehabil 2003;84:1466-72.

9. Borgognone A, Anniboletti T, De Vita F, et al. Ischiatic pressure sores: our experience in coupling a split-muscle flap and a fasciocutaneous flap in a 'criss-cross' way. Spinal Cord 2010;48:770-3.

10. Bertheuil N, Aillet S, Heusse JL, et al. Ischial pressure ulcers: long-term outcome of 2 surgical techniques. Ann Plast Surg 2014;73:686-91.

11. Kim J, Yang HJ. Reconstruction of ischial pressure sores with adjacent muscle primary closure and freestyle perforator-based fasciocutaneous flaps. J Wound Manag Res 2018; 14:85-90.

12. Sharma RK. Split gluteus maximus island flaps for concomitant closure of ischial and sacral pressure sores. Ann Plast Surg 2001;46:52-4.

13. Vincent PL, Pinatel B, Viard R, et al. The gluteus maximus inferior split-muscle flap for the cover of ischiatic pressure ulcers: study of 61 cases. Ann Chir Plast Esthet 2016;61: 845-52.

14. Ramirez OM, Hurwitz DJ, Futrell JW. The expansive gluteus maximus flap. Plast Reconstr Surg 1984;74:757-70.

15. Higgins JP, Orlando GS, Blondeel PN. Ischial pressure sore reconstruction using an inferior gluteal artery perforator (IGAP) flap. Br J Plast Surg 2002;55:83-5.

16. Kim YS, Lew DH, Roh TS, et al. Inferior gluteal artery perforator flap: a viable alternative for ischial pressure sores. J Plast Reconstr Aesthet Surg 2009;62:1347-54.

17. El Omari M, Casoli V, Pinsolle V. Inferior gluteal artery perforator flap: anatomical study for its application in breast reconstruction. Ann Chir Plast Esthet 2011;56:287-97. 